Original Research

\title{
Differences in Muscle Activities and Kinematics between Forefoot Strike and Rearfoot Strike in the Lower Limb during $180^{\circ}$ Turns
}

\author{
Naruto Yoshida' (1) ', Shun Kunugi ${ }^{2}$, Takehiro Konno ${ }^{3}$, Akihiko Masunari ${ }^{4}$, Satoru Nishida ${ }^{5}$, Takashi Koumura ${ }^{6}$, Naoyuki \\ Kobayashi', Shumpei Miyakawa ${ }^{6}$ \\ 1 Department of Acupuncture and Moxibustion, Faculty of Health Care, Teikyo Heisei University, Tokyo, Japan, ${ }^{2}$ Faculty of Liberal Arts and Sciences, \\ Chukyo University, Aichi, Japan, ${ }^{3}$ Hokkaido Nippon-Ham Fighters, Hokkaido, Japan, 4 TOKYO VERDY, Inc., Tokyo, Japan, 5 Faculty of Sports and Health \\ Science, Fukuoka University, Fukuoka, Japan, 6 Faculty of Health and Sport Sciences, University of Tsukuba, Ibaraki, Japan, 7 Faculty of Sport Sciences, \\ Kyushu Kyoritsu University, Fukuoka, Japan \\ Keywords: rearfoot strike, movement system, landing technique, forefoot strike, anterior cruciate ligament \\ https://doi.org/10.26603/001c.23551
}

\section{International Journal of Sports Physical Therapy}

Vol. 16, Issue 3, 2021

\section{Background}

A forefoot strike (FFS) could be a safer landing technique than a rearfoot strike (RFS) during a cutting motion to prevent anterior cruciate ligament (ACL) injury.

\section{Purpose}

This study aimed to determine the joint angles, ground reaction force (GRF), and muscle activity levels associated with FFS and RFS landings during $180^{\circ}$ turns.

\section{Study design}

Cross-sectional study

\section{Methods}

Fourteen male soccer players from the University of Tsukuba football (soccer) club participated in this study. The FFS consisted of initial contact with the toes on the force plates followed by the rearfoot; meanwhile, the initial contact was performed with the heels on the force plates followed by the forefoot for the RFS. Ankle, knee, and hip joint angles were recorded using a three-dimensional motion capture system. GRFs were measured using a force plate. Gluteus medius (GM), rectus femoris (RF), vastus medialis $(\mathrm{VM})$, vastus lateralis (VL), semitendinosus (ST), biceps femoris (BF), tibialis anterior (TA), and lateral gastrocnemius (GL) activities were measured by electromyography.

\section{Results}

The activities of GM, GL, and ST from initial contact to early periods during landing into the ground with the FFS are larger than those with RFS. In addition, the results showed significant differences in lower-limb angles and GRFs between the FFS and RFS.

\section{Conclusion}

These results suggest that there might be differences in ACL injury risk during a $180^{\circ}$ turn between the FFS and the RFS pattern. An investigation into the grounding method that prevents injury is necessary in future studies.

\section{Levels of Evidence}

Level $3 b$

\footnotetext{
a Corresponding author:

Naruto Yoshida, $\mathrm{PhD}$

Department of Acupuncture and Moxibustion,

Faculty of Health Care, Teikyo Heisei University

2-51-4 Higashiikebukuro, Toshimaku, Tokyo 170-8445, Japan

Tel: 81-3-5843-4860; Fax: 81-3-5843-3210

E-mail:naruto-y@kch.biglobe.ne.jp
} 


\section{INTRODUCTION}

Anterior cruciate ligament (ACL) injuries can be broadly divided into two types: contact, which involves physical contact, and non-contact, which does not involve physical contact with another person. Non-contact types account for approximately $70-80 \%$ of all ACL injuries, ${ }^{1}$ and they often occur with motions that induce a change in direction, ${ }^{2}$ sudden deceleration, ${ }^{1}$ and jump landings. ${ }^{1}$ Non-contact ACL injuries are more likely to occur when there is slight flexion and valgus of the knee joint with internal rotation of the tibia. ${ }^{3}$ ACL injuries occur 17-50 msec after landing ${ }^{4}$ and have shown to be associated with an increase in the ground reaction force (GRF), valgus knee angle, and torque within 40 msec of landing. ${ }^{5}$ Moreover, ACL injuries occur when individuals land with the rearfoot or soles horizontal to the ground during deceleration. ${ }^{6}$

The knee valgus angle - a risk factor for non-contact ACL injury - increases when the change-in-direction angle increases ${ }^{7}$ during internally rotated foot landing positions, ${ }^{8}$ when the vertical component of the GRF increases, ${ }^{9}$ and with greater levels of quadriceps activity. ${ }^{1}$ Several studies have examined cutting motions in the diagonal-forward direction. ${ }^{7,8,10}$ With large changes in direction, such as $180^{\circ}$ turns, the direction of movement of the center of gravity is reversed during the deceleration and acceleration phases. In fact, a $180^{\circ}$ turn produces a high risk of injury, similar to a $45^{\circ}$ cut, if performed at the same approach velocity. Scheurs et al. ${ }^{7}$ found similarities in knee abduction moments between $90^{\circ}$ and $180^{\circ}$ turns; however, these joint loads were greater than those produced by $45^{\circ}$ cuts, while Cortes et al. ${ }^{11}$ found greater joint loading with $180^{\circ}$ turns compared to $45^{\circ}$ cuts. Moreover, there are only a few studies that have examined this issue. In particular, few studies have examined $180^{\circ}$ turns in detail, and differences in how foot landing methods affect joint angles, GRFs, and muscle activity have been poorly investigated. Yoshida et al. reported that the forefoot strike (FFS) is less likely to damage the ACL than the rearfoot strike (RFS) during a $60^{\circ}$ cutting motion. ${ }^{12}$ Similarly, Donnelly also reported that a RF fall pattern during unplanned sidestepping places a large mechanical demand on the knee joint, which is associated with an elevated ACL injury risk. ${ }^{13}$ This study aimed to determine the joint angles, ground reaction force (GRF), and muscle activity levels associated with FFS and RFS landings during $180^{\circ}$ turns. Therefore, the authors hypothesize that the FFS reduces the stress on the knee compared with the RFS even during a $180^{\circ}$ turn.

\section{METHODS}

\section{DESIGN}

This cross-sectional study was approved by the Teikyo. Heisei University Ethical Committee (task number 29-022). All procedures were performed in accordance with the Declaration of Helsinki.

\section{PARTICIPANTS}

Participants included 14 male soccer players who were

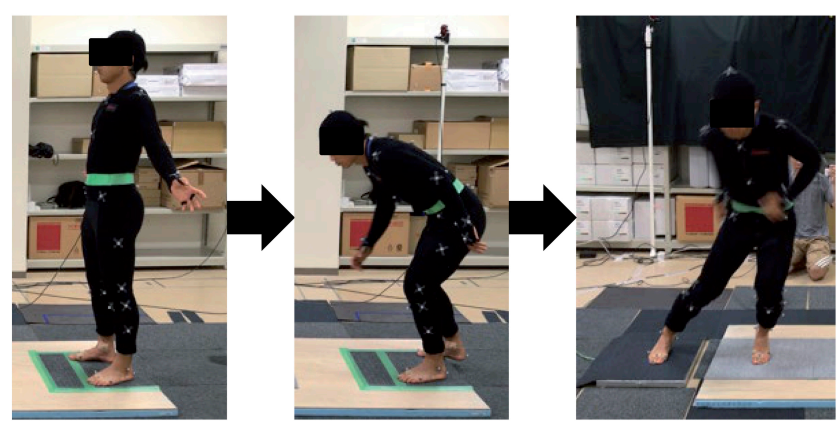

\section{Figure 1: The task motion is a $180^{\circ}$ turn}

(a) Participants standing in the anatomical position. (b) The hip and knee joints slightly flexed, and the right leg is pulled 1 foot-length back. (c) During landing, the right foot is uniformly placed $90^{\circ}$ to the direction of progress to perform a $180^{\circ}$ turn.

members of the university football club (mean height, 169.3 $\pm 5.4 \mathrm{~cm}$; weight, $66.3 \pm 4.6 \mathrm{~kg}$; age, $20.8 \pm 1.0$ years). Players were excluded if they had any injury-related pain or disorder and a history of ACL injury, lower-limb surgery, or severe locomotive organ disease within the prior three months. Each participant provided written or oral consent after receiving a thorough explanation of the study.

\section{PROCEDURES}

The task motion began with the participants standing in the anatomical position with their hip and knee joints slightly flexed and the right leg pulled one foot-length back. Three steps were taken to reach a force platform, approximately $150 \mathrm{~cm}$ away. During landing, the right foot was uniformly placed approximately $90^{\circ}$ in the progress direction to perform a $180^{\circ}$ turn (Figure 1). Landing was performed with an FFS, in which the anterior portion of the foot strikes the platform first, and also in a RFS, in which the posterior portion of the foot strikes the platform first (Figure 2). In other words, the FFS consisted of initial contact with the toes on the force plates followed by the rearfoot; meanwhile, the initial contact of RFS was performed with the heels on the force plates followed by the forefoot like the methods of Cortes. ${ }^{11}$ The participants performed the FFS and RFS, in that order, three times each after being allowed to practice the motions until they were accustomed to them. Failed attempts, described as a landing that was not performed according to the prescribed conditions, were confirmed by foot markers and were discarded.

The participants wore suitable sized motion capture suits, which are suitable clothing for the markers to be placed on the body, with 49 markers attached to the body surface (Appendix 1). The measurements were performed using 12 infrared cameras (OptiTrack, Acuity Inc., Tokyo) and a three-dimensional motion tracking system (Motive Tracker, OptiTrack). The 12 infrared cameras were placed around the force platform to surround the participants while performing each task. Calibration was performed to determine each camera's relative position and the origin and axes of the fixed coordinate system. A calibration wand (CW-500, OptiTrack) was used to obtain a high-quality level of calibration precision. The origin and axes of the fixed co- 
ordinate system were determined using a calibration square (CS-100, OptiTrack), which was placed in the right anterior corner of the force platform from the participants' viewpoint. The right anterior corner of the force platform was the origin. The Z-axis was vertical to the platform $(+)$, the Xaxis was toward the left $(+)$, and the Y-axis was in the posterior direction $(+)$ (right-handed coordinate system). During the task motion, each marker's coordinates were measured using a sampling frequency of $100 \mathrm{~Hz}$ and were then used to calculate the angles of the ankle, knee, and hip using a motion analysis system (Frame-DIASV system, DKH, Tokyo Japan).

The myoelectric potential of the lower extremity musculature was measured using surface electromyography (K800, Biometrics, Newport, UK). Electromyography amplifiers (SX230-1000, Biometrics; inter-electrode distance: $2 \mathrm{~cm}$ ), which function as combined surface electrodes and amplifiers, were placed parallel to the direction of the muscle fibers and fixed from above with elastic tape. A grounding electrode was placed on the left wrist. The induced myoelectric potentials were A/D converted at a 1,000-Hz sampling frequency and saved on a personal computer using the TRAIS system, a data capturing and general-purpose analysis program. Activities of the following muscles were evaluated: gluteus medius (GM), rectus femoris (RF), vastus medialis (VM), vastus lateralis (VL), semitendinosus (ST), biceps femoris (BF), lateral head of the gastrocnemius (GL), and tibialis anterior (TA). Before the task, 5-s data of maximum voluntary isometric contraction (MVIC) for each muscle against manual resistance was measured three times. The greatest value achieved was used as the MVIC in this study. The tasks were performed three times each in the FFS and RFS conditions. The acquired myoelectric potentials were filtered using a band-pass filter (at 20 and 400 $\mathrm{Hz}$ ), and subsequently, a full-wave was rectified. The maximum values of the 3 timed MVICs were averaged, and the myoelectric potentials measured during the tasks were divided by the MVIC to obtain the \%MVIC.

Vertical (Z-axis) and anterior-posterior (Y-axis: corresponding to the $\mathrm{X}$-axis on the motion analysis coordination system) components of the GRF values during the task motion were measured using a force platform (9286BA, Kistler, Kanagawa, Japan) at a $1,000-\mathrm{Hz}$ sampling frequency and saved on a personal computer using the TRAIS system. Participants' GRF values were standardized by dividing them by the body weight. The data were filtered using a low-pass filter at $6 \mathrm{~Hz}$. The point of ground initial contact (IC) was obtained when the vertical component of the GRF reached 10 $\mathrm{N}$. The point at which the GRF was $10 \mathrm{~N}$ or less was defined as take-off. The anterior/posterior component and the lateral component of the GRF were determined by the direction of the body in the landing position. In other words, a starting position to the left was considered to be the same as the forward landing position.

In this study, the joint angle, muscle activity, and GRF values during the FFS and RFS tasks were normalized by the stance phase. The stance phase was defined as that from the IC $(0 \%)$ to the foot-off (100\%) phase. Reports indicate that ACL damage occurs 40 msec after the IC landing, ${ }^{5}$ and the ACL is most loaded at 160 msec. $^{8}$ This study, therefore, defined the early phase as up to $50 \%$ from the IC landing.

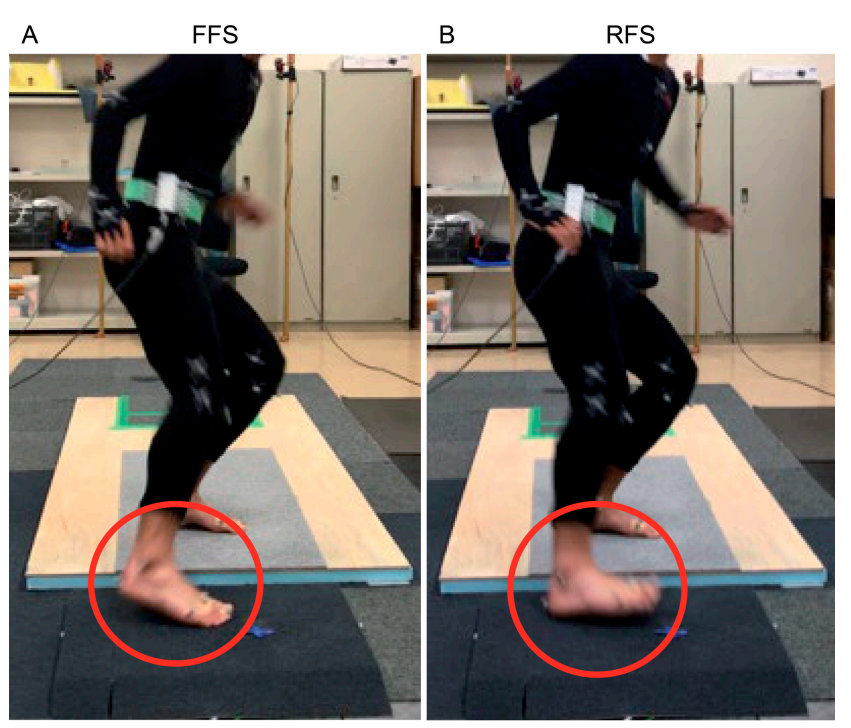

\section{Figure 2: Foot strike landing}

(a) Forefoot strike (FFS), and (b) Rearfoot strike (RFS).

\section{STATISTICAL ANALYSES}

The variables exhibited a normal distribution. To compare RFS and FFS landings, a curve analysis of the data for GRFs, kinematics, and muscle activity was performed using a onedimensional statistical parametric mapping (SPM) twotailed paired t-test. ${ }^{14}$ The statistical analysis of these parameters was performed using MATLAB (version R2019b; Mathworks Inc, Natick, USA). The significance level was set at $\mathrm{p}<0.05$.

\section{RESULTS}

The vertical component of the GRF was significantly larger from $9-20 \%$ and at $99 \%$ with the RFS compared to the FFS $(p<0.05)$ (Figure 3a). The posterior component of the GRF was significantly larger from the IC to $16 \%$ and from $92-100 \%$ with the FFS compared to the RFS ( $p<0.05$ ) (Figure $\underline{3 b})$. The anterior component of the GRF was significantly larger, from $72-81 \%$ with the FFS compared to the RFS $(\mathrm{p}<0.05)$.

GM activity was significantly larger with the FFS than with the RFS from IC to $5 \%(\mathrm{p}<0.05)$ (Figure $4 \mathrm{a})$. ST activity was significantly larger with the FFS than with the RFS from IC to $5 \%(\mathrm{p}<0.05)$ (Figure $4 \mathrm{~b})$. GL activity was significantly larger with the FFS than with the RFS from IC to $14 \%$ $(p<0.05)$ (Figure 4c). There were no significant differences in the activity of the other muscles between the FFS and RFS (Figure $4 \mathrm{~d}-\mathrm{h}$ ).

The hip flexion angle was significantly larger from $18-70 \%$ with the RFS compared to the FFS $(\mathrm{p}<0.05)$ (Figure $\underline{5 a})$. The hip abduction angle was significantly larger from $86-100 \%$ with the RFS compared to the FFS $(\mathrm{p}<0.05)$ (Figure $\underline{5 b})$. The hip external rotation was significantly larger at 95\% with the RFS compared to the FFS ( $p<0.05$ ) (Figure $5 \mathrm{c}$ ).

The knee valgus angle was significantly smaller from $18-34 \%$ with the RFS compared to the FFS $(\mathrm{p}<0.05)$ (Figure 6a). The knee internal rotation angle was significantly 
larger from $14-30 \%$ with the RFS compared to the FFS $(\mathrm{p}<0.05)$ (Figure $6 \mathrm{~b})$. The knee external rotation angle was significantly larger from $86-100 \%$ with the RFS compared to the FFS ( $\mathrm{p}<0.05$ ) (Figure 6b). The plantar flexion angle was significantly larger from the IC to $26 \%$ with the FFS compared to the RFS $(\mathrm{p}<0.05)$ (Figure $7 \mathrm{a})$. The ankle supination angle was significantly larger from the IC to $35 \%$ and from $67-91 \%$ with the FFS compared to the RFS $(p<0.05)$ (Figure $7 \mathrm{~b}$ ). There were no significant differences in the other hip, knee, and ankle angles between the FFS and RFS (Figures $6 \mathrm{c}$ and $\underline{7 c}$ ).

\section{DISCUSSION}

The results of this study indicate that significant differences in the lower-limb angles, muscle activities, and GRFs exist between the FFS and RFS, in varied portions of the stance phase. The larger ankle plantar flexion angle of the FFS demonstrated that the task motion was performed as instructed under the conditions used. ACL injuries occur in the very early phase after landing ${ }^{3}$; therefore, this study considered the different variables produced by the FFS and RFS in the early landing stages. There were significant differences in the vertical and posterior components of the GRFs as well as GM, RF, GL, and ST muscle activation during the early phase as up to $50 \%$ from the IC landing between the FFS and RFS.

The vertical component of the GRF has been shown to be larger with a RFS than with a FFS during two-leg drop jumps. ${ }^{11}$ ACL strain begins to increase during the flight phase before landing and reaches a peak that corresponds to the peak GRF. ${ }^{15}$ Lin used a computer simulation to compare lower extremity kinematics and kinetics between experimental conditions with and without non-contact ACL injuries. ${ }^{16}$ The authors reported that athletes had significantly greater normalized peak posterior and vertical GRF in the simulated injury trials. In addition, Yu reported that the peak vertical ground reaction force significantly correlated with the peak proximal tibia anterior shear force and peak knee extension moment during the landing. ${ }^{17}$ Additionally, compared to a non-injured group, an ACL injury group exhibited larger knee valgus angles, with a vertical GRF component of approximately $20 \%$ larger. $^{2}$ Therefore, the higher vertical component of the GRF observed with an RFS could increase the risk of ACL injury by placing greater tension on the ACL. The vertical component with the FFS was lower than that with an RFS; therefore, a FFS might reduce the risk of ACL injury.

Increased GL activity before landing, which causes plantar flexion of the ankle during the FFS, buffers the impact of landing through eccentric contractions of the GL. Because the plantar flexion angle was significantly larger with the FFS than with the RFS, a FFS might reduce the risk of ACL injury. The hamstrings functions to prevent anterior displacement of the tibia. ${ }^{18-21}$ Because anterior displacement of the tibia increases ACL tension, the current study's results indicate that increased GL and ST activity during the FFS may prevent large amounts of tension from being placed on the ACL, thereby reducing the risk of ACL injury. Activity of the gluteal muscles during landing motions buffers the load on the leg joints in the flexion direction,
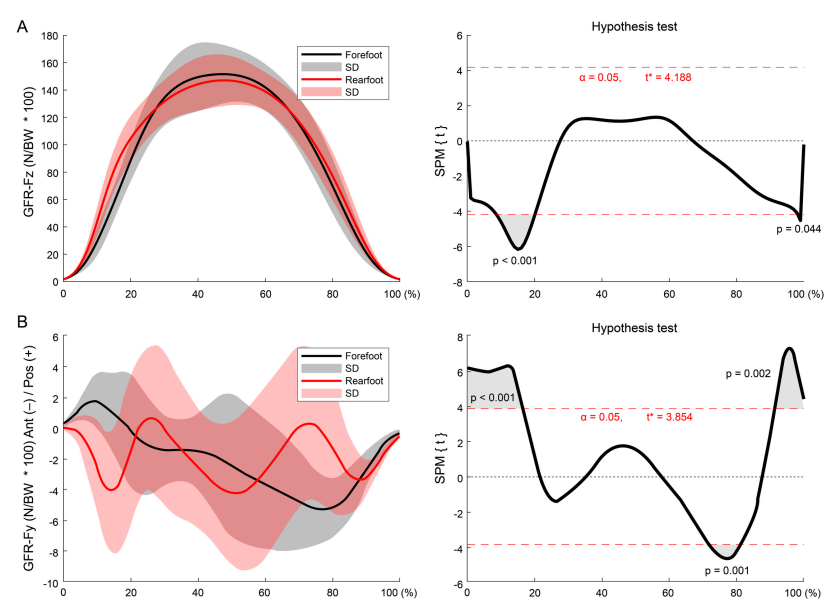

\section{Figure 3: Ground reaction force (GRF)}

(a) The vertical component of the GRF ( $p<0.05)$, and the hypothesis test. (b) The posterior component of the GRF ( $\mathrm{p}<0.05)$, and the hypothesis test. Black line in dicates forefoot, red line indicates rearfoot, and shaded areas indicate standard deviation (SD).

GRF-FY Ant(-)/Post(+): Anterior(-)/ Posterior(+) component of the ground reaction force, N:Newton, BW: Body weight, SPM: statistical parametric mapping.

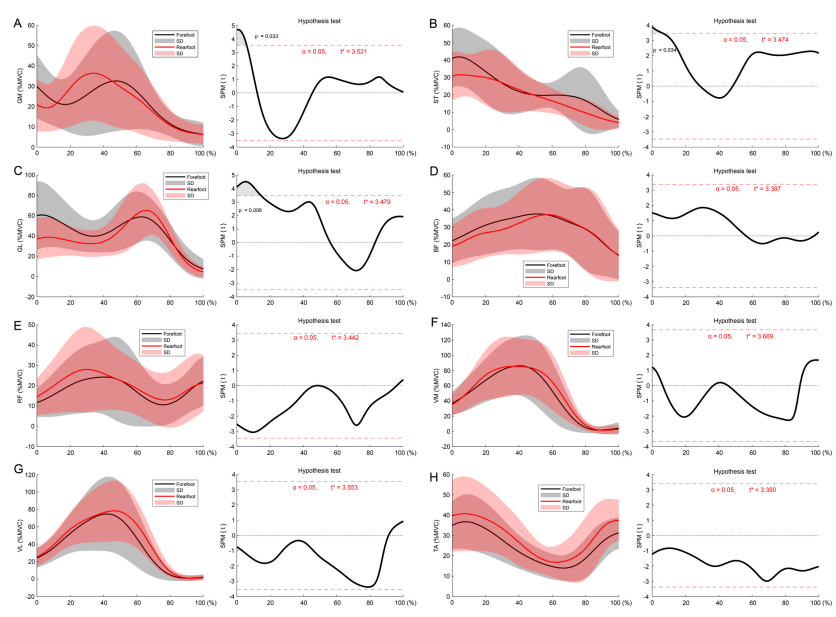

Figure 4: Muscle activity

(a) GM activity, (b) ST activity ( $\mathrm{p}<0.05$ ), (c) GL activity ( $\mathrm{p}<0.05$ ), (d) BF muscles, (e) RF muscles, (f) VM muscles, (g) VL muscles, and (h) TA muscles. Each part of the figure includes a hypothesis test. Black line indicates forefoot, red line indicates rearfoot, and shaded areas indicate standard deviation (SD).

GM, gluteus medius, ST, semitendinosus, GL, lateral head of gastrocnemius, BF, biceps femoris, RF, rectus femoris, VM, vastus medialis, VL, vastus lateralus TA, tibialis anterior, \%MVIC: maximum voluntary isometric contraction, SPM: statistical parametric mapping

helps maintain posture in the sagittal plane, and keeps the pelvis horizontal. These functions stabilize movements of the hip and knee joints, which reduces tension on the ACL. ${ }^{22-25}$ Results of the current study revealed that GM activity increased during the FFS from IC to $5 \%$. Considering that the GM is the primary abductor of the hip joint, this could help maintain the posture in the coronal plane and reduce the risk of ACL injury during $180^{\circ}$ turns.

The knee internal rotation angle increased from $14-30 \%$ with a RFS, which might indicate that the FFS could reduce the risk of ACL injury compared to the RFS. The supination angle of the ankle was larger with a FFS, which may help 
prevent ACL injury, but the risk of ankle sprain should also be considered. The current results also demonstrated that the hip flexion angle was larger, and the knee valgus angle and posterior GRF were smaller with a RFS than an FFS. These results are similar to findings from Cortes et al. stating that the RF landing technique resulted in decreased knee valgus angles, knee flexion, knee adductor moment, posterior GRF, and an increased hip flexion angle at IC. ${ }^{26}$ Jones et al. indicated that the penultimate contact during pre-planned changes in direction helps reduce loading on the turning leg where there is a greater risk of injury to knee ligaments. ${ }^{27}$ Regarding knee valgus, the current study found that the FFS approached the neutral position, and the RFS headed toward the varus direction. The knee valgus angle observed in the current study was small, and there is a possibility that the risk of ACL damage related to the valgus angle has little effect on the RFS and FFS. However, as a proposition to the field, there is a possibility that the RFS is better for actions with a large valgus angle such as the pivot task, similar to findings from Cortes at al. ${ }^{26}$ As for the posterior component of the GRF, even if the anterior component appears in the RFS, if the GRF passes posterior to the center of the knee joint, the tibia receives the anterior pulling force, increasing the risk of ACL damage. On the other hand, even if the rear component is high in the FFS, if the GRF passes anterior to the knee, ACL stress may not increase. However, in the current study, the positions of the penultimate (left) foot and the landing (right) foot were not the same in all trials. Therefore, it is possible to increase the posterior component of the GRF by landing anterior to the body's gravitational center in the FFS; however, the results of the current study do not clarify the reason for the difference in the GRF.

The differences in the hip abduction, hip external rotation, and knee external rotation angles were in the later phase; therefore, it is considered that they would likely have a weak association with the occurrence of ACL injuries.

This study has some limitations. First, examining the biomechanical risk factors cannot precisely reproduce the conditions during which ACL rupture occur, and rely on analyzing a sham movement during the experiment. Although the authors believe that the sham movement/experimental conditions were similar to actual sports activity, there may be some differences in the experimental condition and ACL rupture. Second, the trunk and pelvis should be studied further, because the current study did not measure the angles of trunk side bending and pelvic leaning. Finally, there were only 14 male soccer-playing participants in this study; therefore, there may be errors due to the small sample size. It is also unclear whether the results of this study can apply to female athletes and whether they can be applied to athletes who specialize in sports other than soccer. The difference between the hip joint flexion angle and the posterior component of the GRF may also have been influenced by the lack of evaluation of the penultimate contact. In the future, increasing the number of participants and including athletes of other sports, and including women, will lead to a broader application of the results.
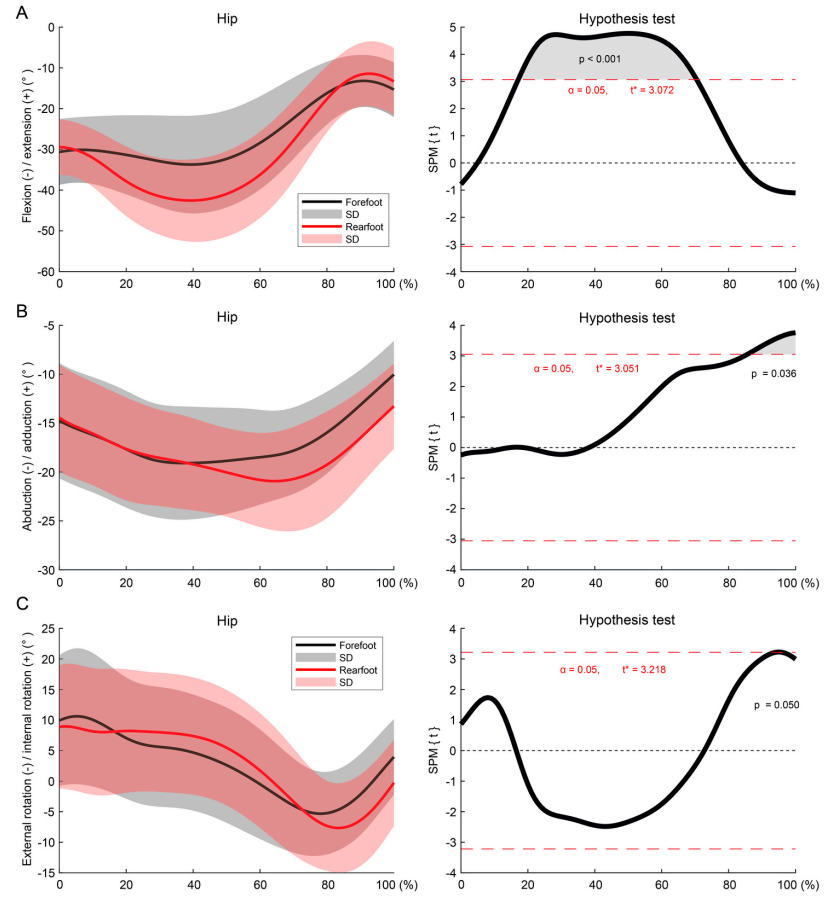

\section{Figure 5: Joint angles of the hip}

(a) Hip flexion angle ( $\mathrm{p}<0.05)$, (b) hip abduction angle ( $\mathrm{p}<0.05)$, and (c) hip external rotation $(\mathrm{p}<0.05)$. Each part of the figure includes a hypothesis test. Black line indicates forefoot, red line indicates rearfoot, and shaded areas indicate standard deviation (SD).

SPM, statistical parametric mapping
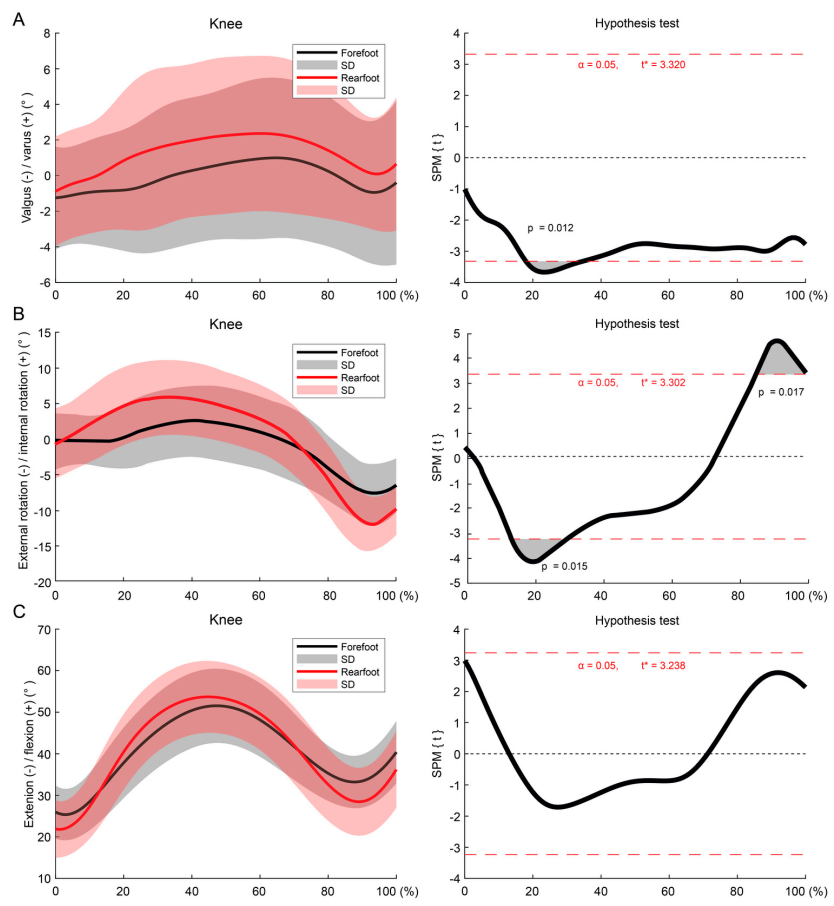

Figure 6: Joint angles of the knee

(a) Knee valgus angle ( $\mathrm{p}<0.05)$, (b) knee internal rotation angle ( $\mathrm{p}<0.05)$, and (c) flexion/extension. Each part of the figure includes a hypothesis test. Black line indicates forefoot, red line indicates rearfoot, and shaded areas indicate standard deviation (SD).

SPM, statistical parametric mapping. 


\section{CONCLUSION}

The present study investigated the difference between the FFS and RFS during $180^{\circ}$ turns. The FFS consisted of initial contact with the toes on the force plates followed by the rearfoot. For the RFS, the initial contact was performed with the heels on the force plates followed by the forefoot. The results of this study indicate that the FFS pattern, when compared to the RFS, produces decreased vertical and GRF components and increased ST muscle activity, implying the potential for reduction in the risk of ACL injury during a $180^{\circ}$-turn movement. Because these results helped clarify that the difference that foot strike has on kinematics and kinetics, it is necessary to further investigate the if a type of foot strike can affect injury prevention.

\section{CONFLICT OF INTEREST STATEMENT}

There are no conflicts of interest for all authors.

Submitted: July 30, 2020 CDT, Accepted: November 26, 2020

CDT
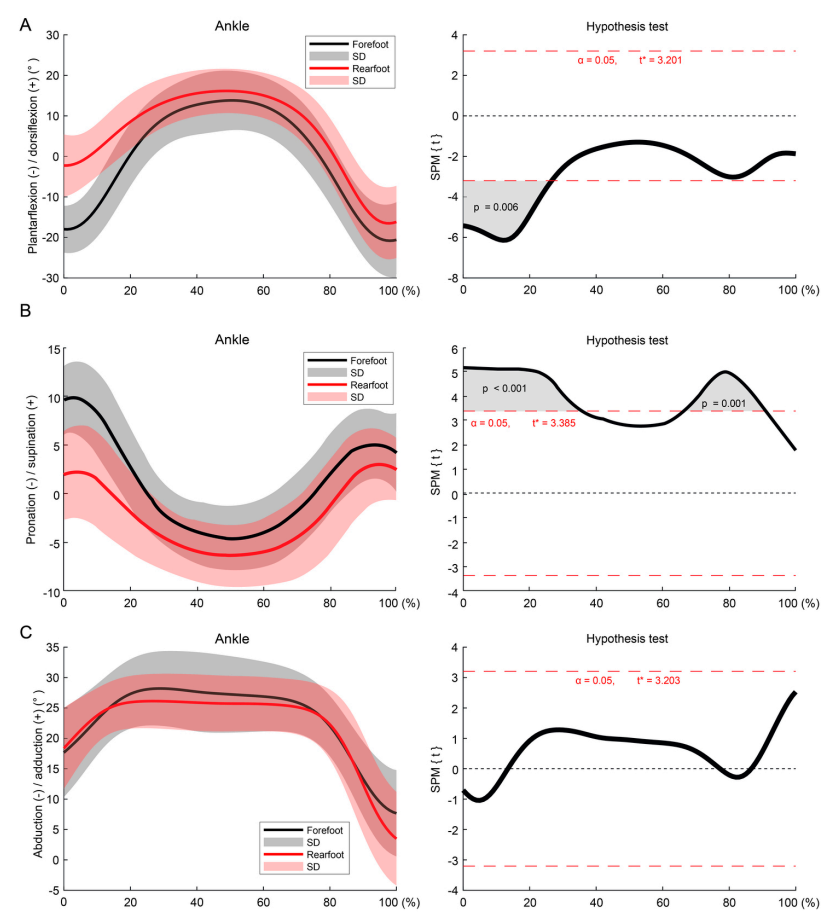

Figure 7: Joint angles of the ankle

(a) The plantar flexion angle ( $\mathrm{p}<0.05)$, (b) the ankle supination angle ( $\mathrm{p}<0.05)$ and (c) adduction/abduction. Each part of the figure includes a hypothesis test. Black line indicates forefoot, red line indicates rearfoot, and shaded areas indicate standard deviation (SD).

SPM, statistical parametric mapping. 


\section{REFERENCES}

1. Boden BP, Dean GS, Feagin JA, Garrett WE. Mechanisms of anterior cruciate ligament injury. Orthopedics. 2000;23(6):573-578. doi:10.3928/0147-7 447-20000601-15

2. Hewett TE, Myer GD, Ford KR, et al. Biomechanical measures of neuromuscular control and valgus loading of the knee predict anterior cruciate ligament injury risk in female athletes: a prospective study. $\mathrm{Am}$ J Sports Med. 2005;33(4):492-501. doi:10.1177/036354 6504269591

3. Bere T, Mok K-M, Koga H, Krosshaug T, Nordsletten L, Bahr R. Kinematics of anterior cruciate ligament ruptures in World Cup alpine skiing: 2 case reports of the slip-catch mechanism. Am J Sports Med. 2013;41(5):1067-1073. doi:10.1177/036354651347934 1

4. Krosshaug T, Nakamae A, Boden BP, et al. Mechanisms of anterior cruciate ligament injury in basketball: video analysis of 39 cases. Am J Sports Med. 2007;35(3):359-367. doi:10.1177/0363546506293 $\underline{899}$

5. Koga H, Nakamae A, Shima Y, et al. Mechanisms for noncontact anterior cruciate ligament injuries: knee joint kinematics in 10 injury situations from female team handball and basketball. Am J Sports Med. 2010;38(11):2218-2225. doi:10.1177/03635465103735 $\underline{70}$

6. Boden BP, Torg JS, Knowles SB, Hewett TE. Video analysis of anterior cruciate ligament injury: abnormalities in hip and ankle kinematics. Am J Sports Med. 2009;37(2):252-259. doi:10.1177/0363546 $\underline{508328107}$

7. Schreurs MJ, Benjaminse A, Lemmink KAPM. Sharper angle, higher risk? The effect of cutting angle on knee mechanics in invasion sport athletes. $J$ Biomech. 2017;63:144-150. doi:10.1016/j.jbiomech.20 17.08.019

8. Sigward SM, Powers CM. Loading characteristics of females exhibiting excessive valgus moments during cutting. Clin Biomech. 2007;22(7):827-833. doi:10.101 6/i.clinbiomech.2007.04.003

9. Hewett TE. Stiff landings are associated with increased acl injury risk in young female basketball and floorball players: Letter to the editor. Am J Sports Med. 2017;45(3):NP5. doi:10.1177/0363546517692761
10. Dempsey AR, Lloyd DG, Elliott BC, Steele JR, Munro BJ, Russo KA. The effect of technique change on knee loads during sidestep cutting. Med Sci Sports Exerc. 2007;39(10):1765-1773. doi:10.1249/mss.0b013 e31812f56d1

11. Cortes N, Onate J, Abrantes J, Gagen L, Dowling E, Van Lunen B. Effects of gender and foot-landing techniques on lower extremity kinematics during drop-jump landings. J Appl Biomech. 2007;23(4):289-299. doi:10.1123/jab.23.4.289

12. Yoshida N, Kunugi S, Mashimo S, et al. Effect of forefoot strike on lower extremity muscle activity and knee joint angle during cutting in female team handball players. Sports Med - Open. 2016;2(1). doi:1 $\underline{0.1186 / \mathrm{s} 40798-016-0056-\mathrm{x}}$

13. Donnelly CJ, Chinnasee C, Weir G, Sasimontonkul $\mathrm{S}$, Alderson J. Joint dynamics of rear- and fore-foot unplanned sidestepping. J Sci Med Sport. 2017;20(1):32-37. doi:10.1016/j.jsams.2016.06.002

14. Pataky TC. One-dimensional statistical parametric mapping in Python. Comput Methods Biomech Biomed Engin. 2012;15(3):295-301. doi:10.10 80/10255842.2010.527837

15. Knudsen B, Fischer MH, Aschersleben G. Development of spatial preferences for counting and picture naming. Psychol Res. 2015;79(6):939-949. do i:10.1007/s00426-014-0623-z

16. Lin C-F, Liu H, Gros MT, Weinhold P, Garrett WE, $\mathrm{Yu}$ B. Biomechanical risk factors of non-contact ACL injuries: A stochastic biomechanical modeling study. J Sport Health Sci. 2012;1(1):36-42. doi:10.1016/j.jsh s.2012.01.001

17. Yu B, Lin C-F, Garrett WE. Lower extremity biomechanics during the landing of a stop-jump task. Clin Biomech. 2006;21(3):297-305. doi:10.1016/j.clinbi omech.2005.11.003

18. Baratta R, Solomonow M, Zhou BH, Letson D, Chuinard R, D’Ambrosia R. Muscular coactivation: the role of the antagonist musculature in maintaining knee stability. Am J Sports Med. 1988;16(2):113-122. $\underline{\mathrm{d}}$ oi: $10.1177 / 036354658801600205$

19. Imran A, O’Connor JJ. Control of knee stability after ACL injury or repair: interaction between hamstrings contraction and tibial translation. Clin Biomech. 1998;13(3):153-162. doi:10.1016/s0268-003 3(97)00030-2 
20. Liu W, Maitland ME. The effect of hamstring muscle compensation for anterior laxity in the ACLdeficient knee during gait. J Biomech. 2000;33(7):871-879. doi:10.1016/s0021-9290(00)0004 $\underline{7-6}$

21. Torry MR, Decker MJ, Ellis HB, Shelburne KB, Sterett WI, Steadman JR. Mechanisms of compensating for anterior cruciate ligament deficiency during gait. Med Sci Sports Exerc. 2004;36(8):1403-1412. doi:10.1249/01.mss.000013579 $\underline{7.09291 .71}$

22. Bobbert MF, van Zandwijk JP. Dynamics of force and muscle stimulation in human vertical jumping. Med Sci Sports Exerc. 1999;31(2):303-310. doi:10.109 7/00005768-199902000-00015

23. Griffin LY, Agel J, Albohm MJ, et al. Noncontact anterior cruciate ligament injuries: risk factors and prevention strategies. JAAOS. 2000;8(3):141-150. do i:10.5435/00124635-200005000-00001
24. McClay Davis I, Ireland ML. ACL research retreat: the gender bias. April 6-7, 2001. Meeting report and abstracts. Clin Biomech. 2001;16(10):937-939. doi:10.1 016/s0268-0033(01)00087-0

25. Zeller BL, McCrory JL, Ben Kibler W, Uhl TL. Differences in kinematics and electromyographic activity between men and women during the singlelegged squat. Am J Sports Med. 2003;31(3):449-456. d oi:10.1177/03635465030310032101

26. Cortes N, Morrison S, Van Lunen BL, Onate JA. Landing technique affects knee loading and position during athletic tasks. J Sci Med Sport.

2012;15(2):175-181. doi:10.1016/j.jsams.2011.09.005

27. Jones PA, Herrington L, Graham-Smith P. Braking characteristics during cutting and pivoting in female soccer players. J Electromyogr Kinesiol. 2016;30:46-54. doi:10.1016/j.jelekin.2016.05.006 


\section{SUPPLEMENTARY MATERIALS}

\section{Appendix 1}

Download: https://ijspt.scholasticahq.com/article/23551-differences-in-muscle-activities-and-kinematics-betweenforefoot-strike-and-rearfoot-strike-in-the-lower-limb-during-180-turns/attachment/60305.docx 